\title{
Enhancing Exercise Performance through Real-time Physiological Monitoring and Music: A User Study
}

\author{
Nuria Oliver \\ Microsoft Research \\ Redmond, WA, USA \\ nuria@microsoft.com
}

\author{
Lucas Kreger-Stickles \\ University of Washington \\ Seattle, WA, USA \\ lucasks@cs.washington.edu
}

\begin{abstract}
We present our findings in using musical feedback to enhance exercise performance by means of a prototype named MPTrain. MPTrain is a mobile and personal system that users wear while exercising. It consists of a set of physiological sensors (heart rate and accelerometer) wirelessly connected to a mobile phone carried by the user. MPTrain's software allows the user to enter a desired workout in terms of desired heart rate stress over time. It then assists the user in achieving the desired exercising goals by: (1) constantly monitoring his/her physiology (heart rate in number of beats per minute) and movement (speed in number of steps per minute); and (2) selecting and playing music (MP3s) with specific features that will guide him/her towards achieving the desired workout goals.

In this paper, we focus on the novel aspects of the MPTrain system and describe in detail our findings from a 9-week runner study, where participants ran with MPTrain for up to four 42minute sessions. The runner study corroborated three hypotheses that we were interested in exploring: The MPTrain system (1) significantly improved the ability of runners to achieve the predefined workout goal, (2) made the experience more enjoyable and (3) increased the runners' perception of the workout's efficacy.
\end{abstract}

\section{INTRODUCTION AND PREVIOUS WORK}

The influence of music in exercise performance has intrigued the research community for quite some time, leading to numerous research studies on the topic [1], [2], [3], [4], [5], [6]. The overwhelming majority of previous work suggests that music has a very positive effect when exercising. A few of the reasons proposed include the idea that music provides a pacing advantage, provides a form of distraction from the fatigue of exercising, affects the mood in a positive way, raises confidence and self-esteem and motivates users to exercise more. Finally, ten different studies agree that exercise endurance, performance perception and perceived exertion levels are positively influenced by music versus non-music conditions [7].

It is, therefore, no surprise that music is often part of the exercise routine for many teens and adults. In particular, MP3 players and heart rate monitors [8], [9] are becoming increasingly pervasive when exercising, especially when walking, running or jogging outdoors. It is not uncommon in the running community to prepare a "running music playlist" [10] that seems to help runners in their training schedules. For example, interval runner Jeff Welch has developed a script which creates an iTunes playlist in which songs stop and start at time intervals to indicate when to switch from running to walking without having to check a watch [11]. Finally, Nike and Apple recently announced their partnership in the NikePod
Sport Kit where the running shoes wireless transmit running pace data to the iPod nano [12] for storage.

However, none of the existing systems to date directly exploits the effects of music on physiology and physical activity in an adaptive and real-time manner. During our background research, we found that all the systems and prototypes developed so far operate in a one-way fashion. They deliver a pre-selected set of songs in a specific order. In some cases, they might independently monitor the user's heart rate or pace, but do not include real-time feedback about the user's state or performance to affect the music selection. The MPTrain system described in this paper addresses these limitations.

MPTrain is a mobile phone based system that takes advantage of the influence of music in exercise performance enabling users to more easily achieve their exercise goals. MPTrain is designed as a mobile and personal system (hardware and software) that users wear while exercising (walking, jogging or running).

In this paper we present several novel aspects of the MPTrain system and report our findings when testing MPTrain with runners. We carried out an 9-week long user study where 20 participants ran with MPTrain for up to four 42-minute long sessions.

The paper is structured as follows: In Section II we briefly describe the MPTrain system. Section III presents MPTrain's music and music selection algorithms. MPTrain's user interface is summarized in Section IV. The user study is presented in detail in Section V. Finally, some conclusions and future directions of research are outlined in Section VI.

\section{MPtrain: A Mobile, Music And Physiology-Based Personal TRainer}

MPTrain is designed as a mobile and personal system (hardware and software) that users wear while exercising (walking, jogging or running). MPTrain's hardware includes a continuous heart rate and acceleration monitor [13] wirelessly connected to a mobile phone carried by the user. MPTrain's software allows the user to enter a desired workout in terms of desired heart rate stress over time. It then assists the user in achieving the desired exercising goals by: (1) constantly monitoring his/her physiology (heart rate in number of beats per minute) and movement (speed in number of steps per minute); and (2) selecting and playing music (MP3s) with 
specific features that will guide him/her towards achieving the desired exercising goals.

MPTrain's algorithms learn the mapping between musical features (e.g. beat), the user's current exercise level (e.g. running speed or gait) and the user's current physiological response (e.g. heart rate). The goal is to automatically select and play the "right" music to encourage the user to speed up, slow down or maintain their pace while keeping him/her on track with the desired workout.

Figure 1 illustrates MPTrain's data flow. The user is listening to digital music on his/her mobile phone while jogging. At the same time, the user's heart rate and speed are monitored and stored on the mobile phone. Feedback from the user's current state (pace and heart rate) is provided to the system, which then compares the user's current heart rate with the desired one according to the current pre-selected workout.

MPTrain's user model is composed of two elements. The (1) next action module determines if the user needs to speed up, slow down or keep their pace of jogging, based on whether his/her heart rate needs to increase, decrease or stay the same. With this information, the (2) music finding module identifies the next song to be played from the music database. Section III explains in detail MPTrain's algorithms for finding the next song to play.

Moreover, MPTrain's interface allows users to check how well they are doing with respect to the desired exercise level, modify the exercising goals or change the music track from the one automatically selected by MPTrain.

In this paper we focus on an evaluation of MPTrain's performance when utilized by runners as part of their running routine. Therefore, we present only the aspects of the system that are relevant to the user study. We also describe in detail the music selection algorithms, as they differ from those previously published.

We shall describe next MPTrain's digital music library (DML) and the music selection algorithms that were utilized in the user study.

\section{Music And Music Selection Algorithms}

MPTrain acts as a personal trainer that uses auditory feedback to encourage the user to accelerate, decelerate or maintain their running pace. The key element is that music improves gait regularity due to the use of the beat, which helps individuals to anticipate the desired rate of movement [14]. The rhythmic structure of the music and the rhythmic actions performed by the body are believed to combine and synchronize.

\section{A. Digital Music Library}

In the user study, the Digital Music Library (DML) was stored in the mobile phone. It contained $70 \mathrm{MP} 3$ songs with durations ranging from $2: 03$ to $5: 55$ minutes, and tempos ranging from 65 to 180 beats per minute. The songs belonged to a variety of music genres and subgenres (e.g. pop, techno, soul, hip hop, etc.), both instrumental and vocal. The DML included additional metadata about each song, such as its

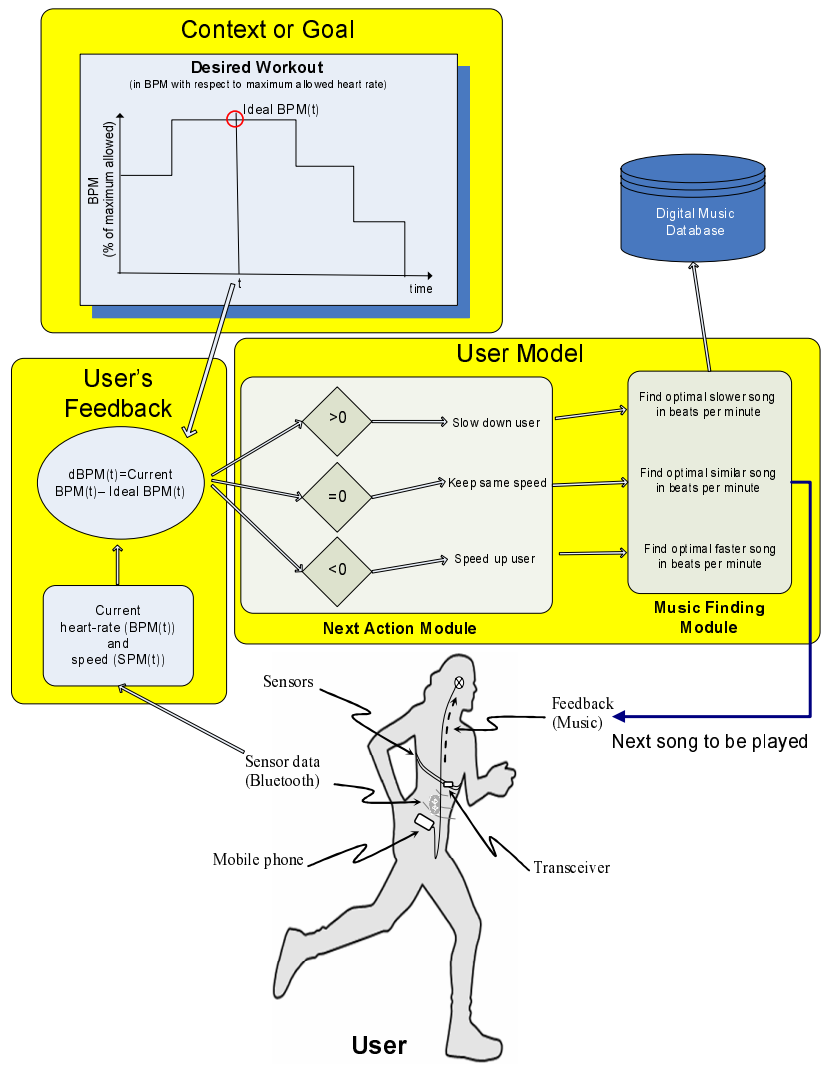

Fig. 1. MPTrain's dataflow.

tempo ${ }^{1}$ and energy in $20 \mathrm{~s}$ window intervals and for the entire song. The music selection algorithms described in this section take into account the song's duration and tempo. Figure 2 depicts three histograms of the average tempo, duration and genres and subgenres of the songs in the DML.

\section{B. Music Selection Algorithms}

MPTrain provides auditory feedback to the user in two different ways: (1) with a metronome and with (2) music.

A metronome is a musical piece that has a steady tempo. The metronomes used in MPTrain are 2 minute long and produce two distinct sounds. A regular "tick" sound to indicate the beat within each measure, and another, distinct "tock" sound that indicates the beginning of each measure. MPTrain utilizes a 4 beats per measure metronome, which sounds: tock tick tick tick tock tick tick tick....

MPTrain automatically determines the tempo of the metronome to be played, ranging from 100 to 220 beats per minute. Note that there are a number of musical features that could have an impact in the user's response to the music, such as the music's actual and perceived tempos, average energy, variance in the energy, emotional factors, etc. The metronome

\footnotetext{
${ }^{1}$ Determined automatically or manually.
} 

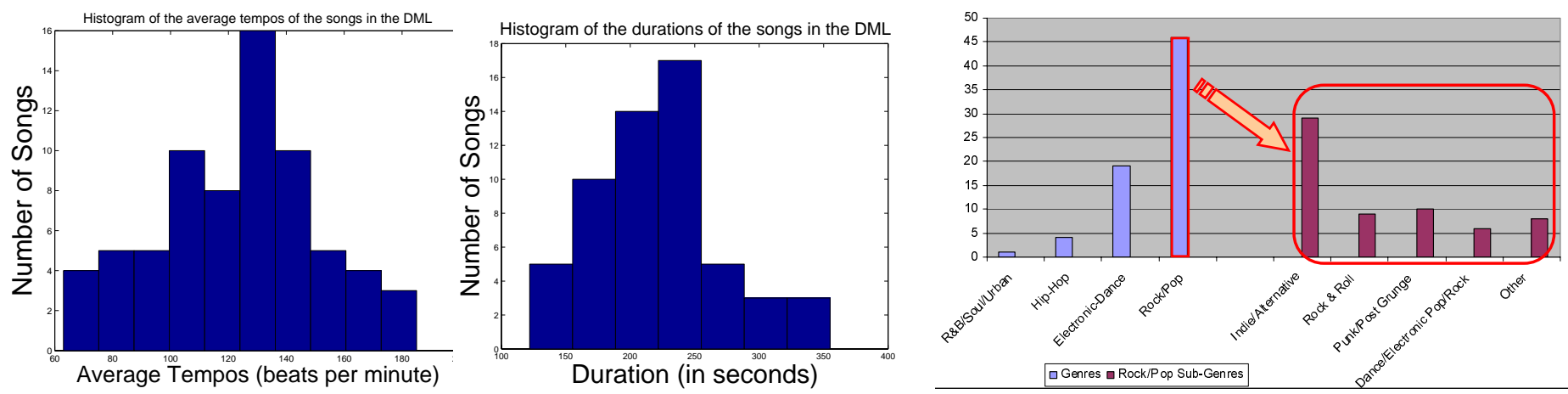

Fig. 2. Histograms, from left to right, of the average tempo, duration and genres and subgenres of the songs in the DML

mode allows us to isolate the tempo from all other factors in the music and therefore to achieve a better understanding of the impact of the tempo in the runner's pace.

We shall describe next MPTrain's algorithms for selecting the metronome's tempo or the music to play. Figure 3 illustrates MPTrain's music selection behavior on some example data. The top graph in the figure depicts the user's heart rate (in red) and the desired heart rate (in blue). It also highlights 4 heart rate zones, namely, from bottom to top: (a) weight management, (b) aerobic, (c) anaerobic and (d) overexertion[15]. The workout in the user study described in this paper (as shown in Figure 6) contained weight management, aerobic and anaerobic regions. In addition, MPTrain assisted runners in avoiding over-exertion. The bottom graph contains the runner's actual pace (in green) and the metronome or song's tempo (in magenta). There are 5 regions, depending on the runner's pace, ranging from walking to sprinting.

In its current implementation, MPTrain does not take any action until one of these three conditions is true:

1. There are a few seconds left (e.g. 10) before the end of the current song ${ }^{2}$ : In this case, MPTrain determines whether the user needs to increase, decrease or keep the running pace, by comparing the user's average heart rate during a time window of the past $N$ seconds (where $N$ is typically 25) with the desired heart rate from the desired workout for that day. Once it has determined the action to take, it searches the user's digital music library (DML) for the optimal song to play (or, in the case playing back a metronome, searches for the optimal metronome).

Depending on the situation, MPTrain will look for a song whose beat is similar, higher or lower than that of the song currently being played, according to the difference between the actual and desired heart rates. In the event that the workout target is about to change (e.g. within 20 seconds), MPTrain selects a song appropriate to the next workout target.

Region 2 in Figure 3 illustrates this behavior. In the top graph of the Figure, the user's average heart rate (in red) over

${ }^{2}$ In the following, we will use the term song to indistinctively refer to an actual song from the DML or a metronome of a particular tempo.

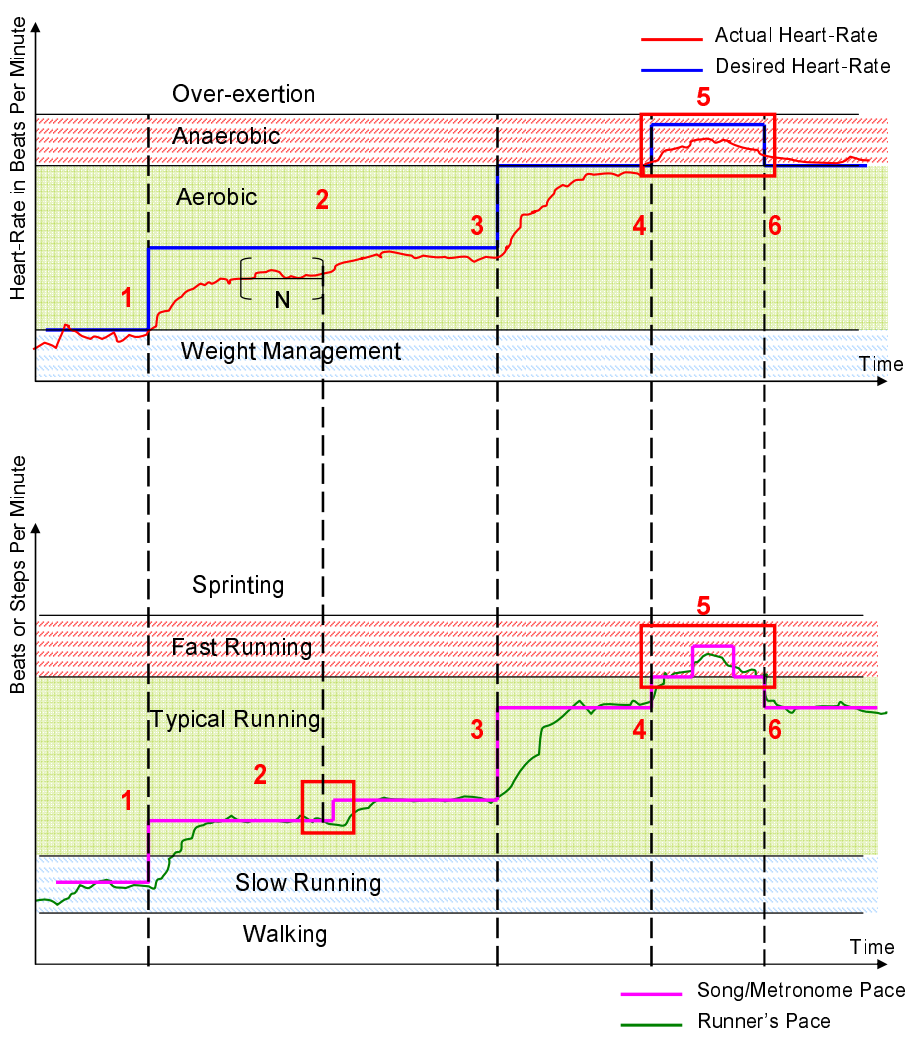

Fig. 3. MPTrain's music selection behavior.

the past $N$ seconds has been lower than the desired heart rate (in blue). As the user has been closely tracking the current song's tempo (Region 2 in the bottom graph in the Figure), MPTrain selects a song whose tempo is higher than that of the current song. The increase in beat in the song is proportional to the percentage of error between the user's actual and desired heart rates. At the same time, MPTrain respects the user's physical limitations and past behavior by setting an upper limit (e.g. $5-10 \%$ ) on the increase of beat for the next selected song. As the song's tempo increases (right part of Region 2 
in the bottom graph), so does the runner's pace, causing his heart rate to increase (right part of Region 2 in the top graph) and consequently approach the target heart rate.

A typical workout would target paces in the typical running region (e.g. 140 to 170 steps per minute). Paces slower or faster than those are treated differently by the system. MPTrain will select songs with tempos below or above the typical running region only if (1) there is a need to decrease or increase the user's heart rate, and (2) the user's pace has been closely tracking the song's tempo for the past $N$ seconds (where $N$ is typically 10). For example, in Region 5 of the Figure (top graph) the user's heart rate is below the desired heart rate. Therefore MPTrain tries to speed up the user. It first selects a song whose tempo is at the upper limit of the typical running region (left segment of Region 5, bottom graph). As the user's heart rate is still too low but his pace has closely tracked the song's tempo, MPTrain selects next a song whose tempo is higher than the current song's tempo (middle segment of Region 5, bottom graph), entering the fast running region. The user's heart rate increases accordingly but the song's tempo seems to be too fast for the runner to keep track. Therefore, in the next song change (right segment of Region 5, bottom graph), MPTrain selects a song slower than the previous one, hoping that the user will be able to synchronize to its tempo again.

This is an important phenomenon to note. Our experimental data supports the following intuitive observation: When a song's tempo is significantly different than the user's pace, it is very hard for the system to induce pace changes in the user and therefore affect the user's heart rate. However, when the song's tempo is within a range (e.g. 5 to $15 \mathrm{bpm}$ ) of the user's pace, it is very likely that the user will synchronize his/her movement to the music, significantly increasing the ability of the music to influence the user's pace and therefore heart rate.

2. There is a discontinuity in the desired workout pattern, such as moving from a warming-up (about $60 \%$ of maximum heart rate reserve ${ }^{3}$ ) to a weight management section (about $70 \%$ of maximum heart rate reserve) in the desired workout. This behavior is illustrated in regions 1, 3, 4 and 6 of the Figure. In this case, MPTrain interrupts the song that is currently playing, unless the song has been playing for a very short time (e.g. less than $30 \mathrm{~s})^{4}$. MPTrain selects the next song to play as described in case (1) above, taking into account the error between the runner's heart rate and the desired heart rate, and the relationship between the runner's pace and the song's tempo.

3. The user explicitely requests a change of song. In this case, MPTrain selects a different song from the DML whose features still satisfy the constraints given the situation.

MPTrain's current implementation uses two empirically

\footnotetext{
${ }^{3}$ As given by Equation 1 .

${ }^{4}$ In this case the song has already been selected based on the new target.
}

learned functions to map the the influence of the music's beat on the running pace, and the user's physiological response to that pace. This model is used to make a statistically accurate track selection. Further versions will also incorporate historic information about the user's past performance and responses to each of the songs.

\section{USER's INTERFACE}

MPTrain's software is implemented as a Windows Mobile application, with all its modules (sensor data reception, data analysis, display, storage and music selection and playback) running simultaneously in real-time on the mobile phone.

Figure 4 shows MPTrain's main interface window. The solid graph in the center of the window depicts the desired workout pattern for that day. It consists of a graph of the desired workout heart rate (y-axis) - in \% of the user's heart rate reserve (see Equation 1) - over time (x-axis). For example, the depicted workout in the Figure contains a warm-up period (left-most part of the graph), followed by some intense and moderate running periods (middle parts of the graph), and ended by a cool-down phase (right-most part of the graph).

When MPTrain is in operation, a line graph is superimposed to the desired workout depicting the user's actual heart rate. This allows the user to compare in real-time his/her performance to the desired one. The user can easily specify his/her desired workout by either selecting one of the pre-defined workouts or creating a new one (as a simple text file). At the bottom of the Figure there is the name of the song currently being played, the total time of workout since the user started running, and the amount of time that the current song has been playing for. On the top of the Figure, there is the \% of battery life left on the sensing module, the user's current pace and heart rate, the total number of steps and the total number of calories burned. In addition, the user can access this information in the form of audio feedback by just pressing one button on the phone. Finally, the interface also allows the user to pause/resume their workout at any time, and to skip songs if desired. MPTrain logs all user's actions. Later versions of the system will incorporate these logs in the music selection algorithms.

\section{USER EVALUATiON}

To validate MPTrain, we carried out a 9-week user study. Participants were amateur runners who ran with the MPTrain system for up to 4 running sessions of 42 minutes long each.

\section{A. Hypotheses}

The ability to achieve a predefined workout goal should be higher when running with the songs or metronome as selected by MPTrain, than when running without any music or with music randomly selected. It is hypothesized that:

1) Runners listening to MPTrain's selected music or metronome are able to track the predefined workout goal better than those running without any music or with randomly selected music. 


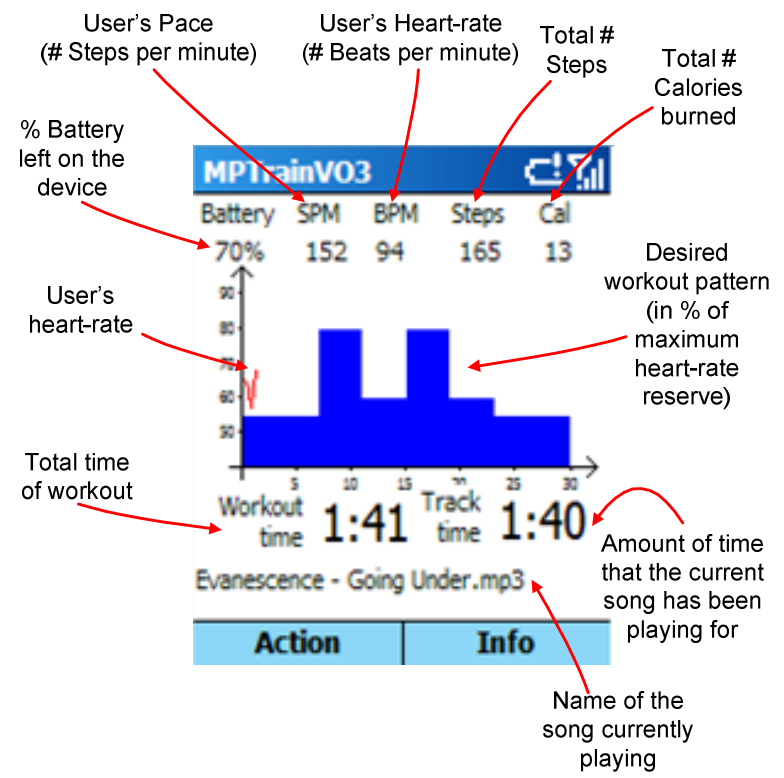

Fig. 4. Main window of MPTrain's User Interface, displaying the user's current heart rate, running pace, total number of calories burned, song being played and desired workout pattern (in blue).

2) Runners enjoy their workout more when listening to MPTrain's music than when running without music or with randomly selected music.

3) Runners perceive their run to be more effective towards reaching the workout goals when running with MPTrain's auditory feedback than when running in silent or random modes.

\section{B. Measures}

1) Task Performance: Task performance was measured by the percentage of time that the user's percentage heart rate reserve (see Equation 1) was within a $[-5,+5] \%$ range of the target percentage of heart rate reserve, as it is commonly defined in the sports literature [16], [17].

Target heart rate training is known in the sports literature as a systematic method of improving cardiovascular fitness. Target heart rates are typically expressed in percentage of the heart rate's reserve, defined by:

$$
\text { PHR } R_{\text {reserve }}=\left(H R_{\text {max }}-H R_{\text {res }}\right) * P+H R_{\text {res }}
$$

where $P H R_{\text {reserve }}$ is the percentage $(\mathrm{P})$ of heart rate's reserve and $H R_{\max }$ and $H R_{\text {res }}$ are the maximum and resting heart rates respectively.

In addition, the vast majority of personal training systems define target "zones". Because of the natural fluctuations of the human heart rate, it would be virtually impossible otherwise to maintain any pre-selected heart rate. Moreover, heart rate, as most physiological signals, is relatively slow changing and therefore needs a few seconds to reach a specific range of values.

Figure 5 illustrates the target zones on a detailed section of the desired workout utilized in the study. It also displays the heart rate of one of the participants on three running conditions

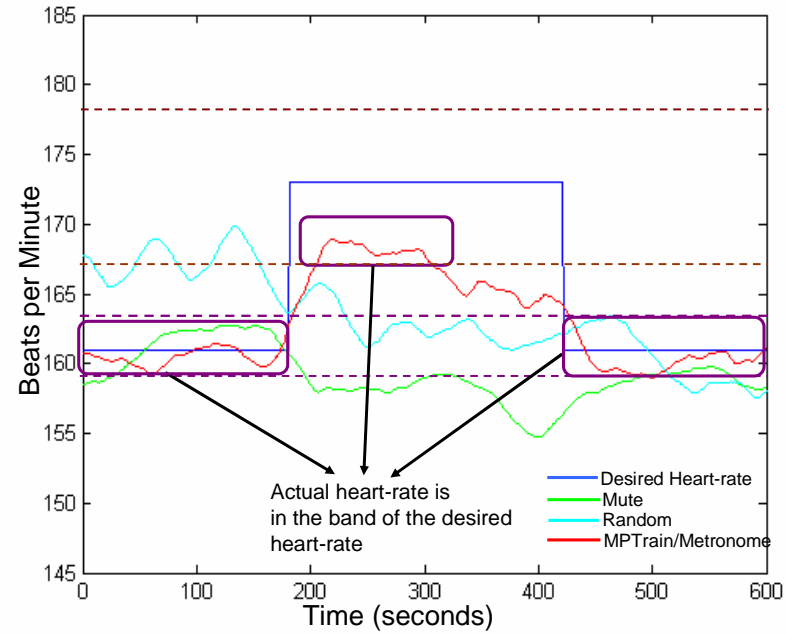

Fig. 5. Detail of the heart rate zones defined for 2 of the workout regions. The Figure highlights the areas where the runner's heart rate is within the band for each of the running conditions.

(mute in green, random in cyan and MPTrain/Metronome in red). Note how the runner's heart rate falls within the target zone for a significant percentage of time only in the MPTrain/Metronome condition.

2) Enjoyment: The runner's enjoyment of the workout was assesed by a post-run questionnaire that explicitely asked the runners to (1) rate their run on a scale from 1 to 10 in terms of enjoyment (with 1 minimum and 10 maximum enjoyment respectively); and (2) compare their run to previous runs with and without the system.

3) Perceived Usefulness: The post-run questionnaire asked runners to rate the run's effectiveness towards achieving the workout goal, on a 1 to 10 scale. It also asked participants to rate the run's perceived efficacy when compared to previous runs with and without the system.

4) Design: A factorial within-subject design with one independent variable, named condition, was used. The variable referred to the four sessions in the study: running without music (mute condition), with random music (random condition), with MPTrain's selected music (MPTrain condition) and with a metronome (Metronome condition). The first run was in the mute condition for all participants. This allowed participants to get used to the system's hardware and software. In addition, it allowed us to obtain a good estimate of the participant's resting and maximum heart rates.

The resting heart rate was computed in the office, when the participants came to pick up the system. The maximum heart rate was automatically computed by MPTrain by means of the standard Miller [18] formula ( $\mathrm{MaxHR}=217-(0.85 \times a g e)$ ). However, this formula does not take into account personal differences due to factors such as fitness level, gender, resting heart rate, body weight, altitude and true maximal exertion. Therefore, we used the data from the mute run to estimate each participant's actual maximum heart rate.

The order for the rest of the running conditions was ran- 
domly assigned to participants, to compensate for any potential order effects.

In the remaining of the discussion we will refer to the runs with auditory feedback as MPTrain/Metronome, without distinguishing between them. We shall present quantitative and qualitative differences between them in Section V-C.2.

5) Test System: All participants used the same hardware and software. The hardware consisted of an AliveTec ECG and acceleration monitor [13] attached to either a leather chest-band that contained the 2-lead ECG sensors or to 2 adhesive ECG electrodes by Ambu, which are designed for ambulatory recording. The sensors were wirelessly connected to an Audiovox SMT5600 mobile phone running MPTrain's software. The Digital Music Library was stored in the phone and was the same for all runners, as described in Section III. Runners were offered an armband pouch to carry the mobile phone during their run. About $50 \%$ of the runners were interested in visually tracking their performance through the run and therefore chose to carry the phone in their hand instead of carrying it in the armband.

6) Task: Participants were asked to run for 42 minutes. They were asked to try to achieve as closely as possible the workout pattern that appeared on the phone's interface and is depicted in Figure 6. The workout consisted of a 5-minute period of warming-up at $65 \%$ of heart rate reserve, three 8 minute periods at $75 \%$ of heart rate reserve with two 4 -minute intervals at $85 \%$, ending with a 5 -minute cool-down phase at $65 \%$.

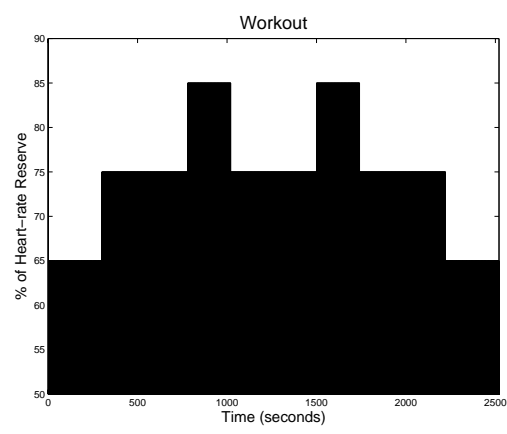

Fig. 6. Workout pattern used in the runner study.

Moreover, all participants were asked to run on the same trail for all of the runs in the study, and preferably on a flat surface. Note that the current version of MPTrain does not account for incline on the terrain. Therefore, all changes in the runner's heart rate are assumed to have been caused by changes in the runner's pace.

7) Procedure: Participants took part in up to 4 running sessions on 4 separate days. Each session corresponded to a running condition: (1) mute or without music; (2) random or with music randomly selected; (3) metronome or with a metronome as selected by MPTrain; and (4) MPTrain or with music as selected by MPTrain.

Before their first session, participants filled out an online questionnaire to record personal data and attributes, including their exercise and running experience.

The first session started with instructions and a demonstration on how to use the MPTrain system. They were asked to put the system on in order to verify that the hardware was properly working and to compute their resting heart rate. Use of MPTrain's user interface was then explained and demonstrated. The goal of the study was emphasized during this set-up period: they were supposed to achieve the workout pattern that appeared on the phone's interface and is depicted in Figure 6. Once they were confident with how to use the system (typically after 5 or 10 minutes), they were sent off for a 42 minute run in mute mode (e.g. without any music).

After the run on mute mode was finished, they returned the system to us and were asked to fill out an online post-run questionnaire for the mute condition.

The running conditions for the rest of the sessions were randomized. After each session, they were asked to fill out the corresponding online post-run questionnaire.

To further motivate the runners to achieve the workout goals, we offered a $50 \$$ gift certificate to the runner whose heart rate best tracked the desired workout heart rate in any of the running conditions.

8) Participants: From an initial pool of 36 participants who registered for the study, 20 (13 males and 7 females) did at least one run with the system. They were recruited by email advertisement to several groups within a very large corporation. All participants were regular runners of various levels of expertise and fitness. The average number of times per week that they run was 4 for an average of 52 minutes each time.

Thirteen participants ( 9 males and 4 females) completed at least three runs with the system, whereas 7 only finished one or two runs due to traveling, injury (not related to the user study) or sickness.

The average age of participants was 36, ranging from 24 to 63 years old. About $35 \%$ of participants typically run with other people ( 7 participants), $60 \%$ had ever worn a heart rate monitor while running (12 participants), but only $40 \%$ wore one regularly ( 8 participants). $60 \%$ of participants listened to music regularly while exercising. The two main reasons why they reported that they listened to music were because music "helped pass time faster" ( $91.7 \%$ or 11 participants) and it "helped them maintain a certain pace" $(83.3 \%$ or 10 participants). For those who did not run with music (8 participants), the most commonly cited reason not to run with music was that "the music device was too cumbersome" (62.5\% or 5 participants).

\section{Results}

1) Data Analysis: Table I summarizes the quantitative analysis of the data. Note that the Table contains the average results for the MPTrain and Metronome modes, in order to better understand the impact of providing auditory feedback to the user versus not. The differences between the MPTrain and Metronome modes appear in Table II. Finally, the highlighted cell on each row corresponds to the best condition. 
TABLE I

PERFORMANCE UNDER EACH OF THE RUNNING CONDITIONS

\begin{tabular}{|c|c|c|c|}
\hline & \multicolumn{3}{|c|}{ Running Condition } \\
\hline & Mute & Random & MPTrain/Metronome \\
\hline $\begin{array}{c}\text { Mean \% Time } \\
\text { HR in Range }\end{array}$ & 42.6 & 41.7 & $\mathbf{5 4 . 0}$ \\
\hline $\begin{array}{c}\text { Mean HR Error } \\
\text { (\% of reserve) }\end{array}$ & 5.1 & 4.1 & $\mathbf{2 . 5}$ \\
\hline $\begin{array}{c}\text { Mean \% Time } \\
\text { SPM in Range }\end{array}$ & N/A & 7.0 & $\mathbf{3 3 . 1}$ \\
\hline Best Mode (\%) & 30.8 & 7.7 & $\mathbf{6 1 . 5}$ \\
\hline Best SPM (\%) & N/A & 11.1 & $\mathbf{8 8 . 9}$ \\
\hline
\end{tabular}

TABLE II

Performance of the MPTrain versus Metronome condition

\begin{tabular}{|c|c|c|}
\hline & \multicolumn{2}{|c|}{ Running Condition } \\
\hline & MPTrain & Metronome \\
\hline Mean \% Time of HR in Range & 45.7 & $\mathbf{6 2 . 3}$ \\
\hline Mean HR Error (\% of reserve) & 3.2 & $\mathbf{1 . 8}$ \\
\hline Mean \% Time SPM in Range & 25.6 & $\mathbf{4 0 . 6}$ \\
\hline Best Mode (\%) & 23.1 & $\mathbf{3 8 . 5}$ \\
\hline Best SPM (\%) & 33.3 & $\mathbf{5 5 . 6}$ \\
\hline
\end{tabular}

The first row of Table I contains the mean percentage of time that the runner's percentage of heart rate reserve was within a $[-5+5] \%$ band of the desired percentage of heart rate reserve. The second row contains the mean error of the runner's heart rate when compared to the desired heart rate (in percentage of heart rate reserve). The mean percentage of time that the runner's pace (in steps per minute or SPM) was within a $[-5+5] \%$ band of the desired tempo (in beats per minute) appears on the third row. The percentage of time that each mode was the best mode - as measured by how well it helped the runner achieve the workout goal - is summarized on the fifth row. Finally, the last row contains the percentage of time that the runner's pace best matched the desired tempo, for each of the running conditions.

As can be seen on the table, the MPTrain/Metronome condition was the best in all cases, i.e. it caused runners to spend more time in the target heart rate zone and with the target pace.

2) MPTrain's Music vs Metronome: There were, however, significant quantitative and qualitative differences between the MPTrain and Metronome modes ${ }^{5}$. In fact and purely from a quantitative perspective, the Metronome mode was superior to MPTrain in all quantitative measures, as depicted in Table II.

There are a few reasons why runners performed better under the Metronome than under the MPTrain condition: (1) All participants ran with the same music, instead of their personal music collection. In consequence, some participants were more familiar with the songs than others and some enjoyed the music selection more than others. This had an impact on how well they could identify the songs' tempos. On the other hand, all runners were able to quite easily identify the metronome's tempos; (2) There was a certain level of emotional response to the music that was non-existent with the metronome; (3)

${ }^{5}$ From the thirteen runners that ran on MPTrain, only 10 also ran with the Metronome.
As described in Section V-C.4, about $20 \%$ of the runners were unable to correctly identify the tempo of the songs some of the time. Therefore, they synchronyzed their pace to their perceived tempo rather than the actual tempo, leading to an undesired behavior. This phenomenon never occurred with the metronome.

In contrast (see Section V-C.5 and Table III), running with the MPTrain condition was significantly more enjoyable than running with the Metronome.

3) Personal Differences: MPTrain is designed to be a highly personal and adaptive system. However, for the purpose of the study, we asked all participants to do the same workout routine and to listen to music from the same DML. In consequence, there were quite some personal differences from participant to participant.

Figure 8 depicts the task performance for the thirteen participants who completed the runs under the mute, random and MPTrain and/or Metronome conditions. On the top graph, the $X$-axis corresponds to the subject number and the $Y$-axis to the percentage of time that the runner's heart rate was within the target zone. The bottom graph depicts the percentage of time that the runner's pace was within the target tempo. The Figure highlights 3 different kinds of individuals, based on their response to MPTrain's auditory feedback:

1) About $50 \%$ of the runners responded very well to MPTrain's style of coaching via auditory feedback. They adjusted their pace as needed and, in consequence, their heart rates tracked significantly better the desired workout than in the conditions without any feedback (mute and random). Arrow 1 on the Figure points to an exemplary runner belonging to this group.

2) About $30 \%$ of the runners adjusted their pace as needed, but not enough to achieve a significant improvement with respect to the mute and random conditions. We believe that runners in this group would perform increasingly better as they become more used to MPTrain's style of coaching and to the specific music libray in the phone. Interestingly and in a few cases, runners in this group adjusted their pace incorrectly. For example, when the system was cueing them to run faster, they would slow down and vice-versa. We shall delve on this topic later. Arrow 2 points to an exemplary participant from this group.

3) Finally, a small percentage of runners (about 20\%) did not change their running pace when cued by the system. They seemed to be so accustomed to their running habits and workout that they did not deviate from them during the study, even though they knew that they had a specific workout goal to achieve. Arrow 3 illustrates an exemplary runner in this group.

Figures 7 and 9 illustrate the impact of MPTrain's auditory feedback on two different runners.

Figure 7 shows the performance of one runner under the mute, random and MPTrain/Metronome conditions. The red line corresponds to the runner's heart rate over time and the blue line represents the desired workout targets (expressed in 

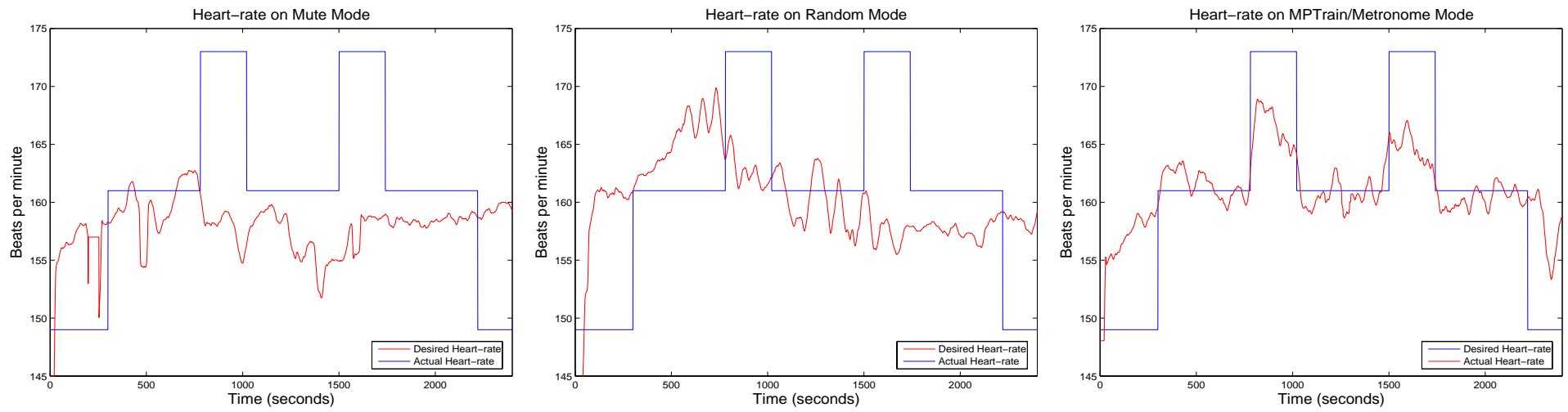

Fig. 7. Desired (blue) and actual (red) heart rate for a runner on mute (left), random (middle) and MPTrain/Metronome mode (right). Note how in the MPTrain/Metronome condition the runner's heart rate follows the desired heart rate.

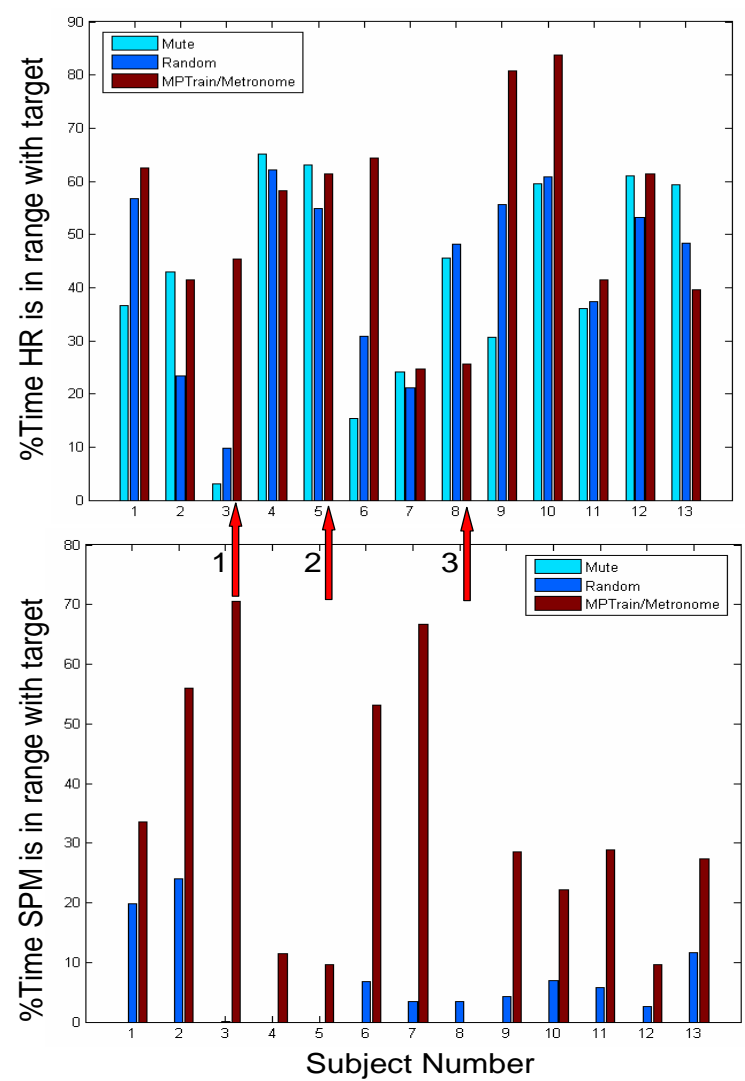

Fig. 8. Top Graph: Percentage of time spent within a $5 \%$ band of the target heart rate for 13 runners, where cyan corresponds to mute mode, blue to random mode and maroon to MPTrain/Metronome mode. Bottom Graph: Percentage of time spent within a $5 \%$ band of the target tempo for the same 13 runners, where cyan corresponds to mute mode, blue to random mode and maroon to MPTrain/Metronome mode.

heart rate - beats per minute -). As can be seen in the Figure, the runner's heart rate nicely tracks the desired heart rate only in the MPTrain/Metronome condition, being unable to do so in any of the other conditions. The impact of the auditory feedback on this runner is very significant.

Figure 9 depicts the response of a different runner to the Metronome and it illustrates the impact that the running pace has on the runner's heart rate. The top graph illustrates the runner's heart rate (in red) and desired heart rate (in blue) over time. The bottom graph contains the runner's pace (in blue) and the metronome's tempo (in green). Note how the runner's pace closely adjusts to the metronome's tempo. This causes his heart rate to increase or decrease accordingly. In consequence, the runner's heart rate tracks the desired workout heart rate very well.
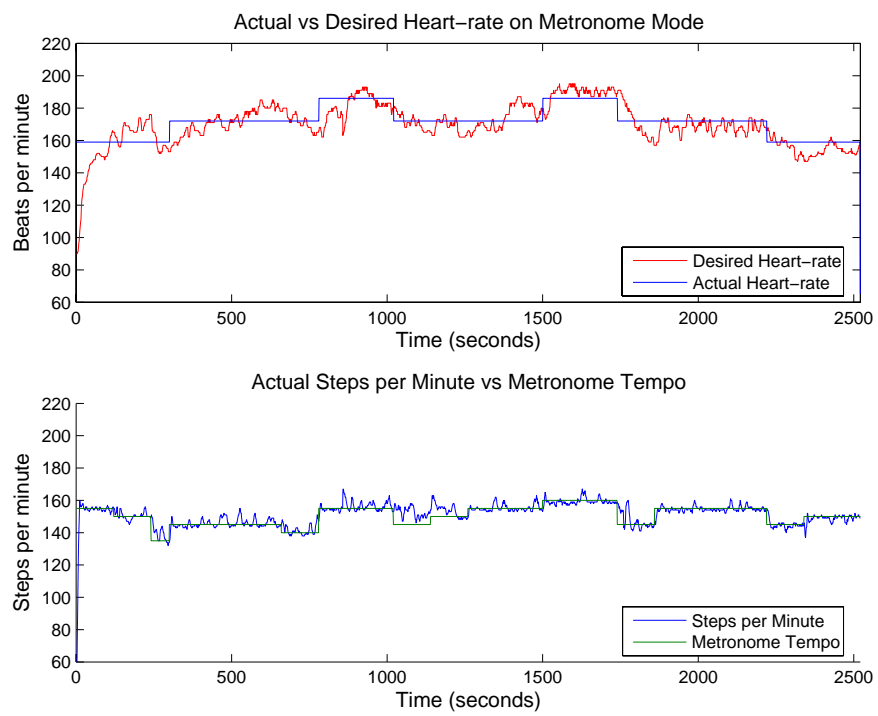

Fig. 9. Top Graph: Desired (blue) and actual (red) heart rate for a user running on Metronome mode. Bottom Graph: Metronome's tempo (green) and user's pace (blue) in steps per minute. Note how well the user is able to track the metronome's tempo with his pace. In consequence, his heart rate nicely tracks the desired heart rate.

4) Perceived Song Speed Study: As mentioned above, there were significant personal differences regarding how the participants responded to the music provided by MPTrain.

A large percentage of participants (about $50-55 \%$ ) were able to identify and synchronize with the major beat of the music. These runners typically tracked the target workout very well.

Other participants (about $15-20 \%$ ), however, seemed to be 
so ingrained in their running habits that they simply ignored the music and other cues intended to assist them in reaching the workout targets. As the target workout was new to them, they generally found it hard to deviate from their well acquired running routine.

Finally, a third group (about 30\%) did change their running pace as prompted by the system. However, they seemed unable to correctly identify the actual beat of some of the songs. Instead, they tried to speed up or slow down, based on whether they perceived the most recently selected song to be faster or slower than the previously selected song.

Among this final group of loose trackers a strange phenomenon was noted. On occasion, the runner would respond in a way that was the opposite of what was intended. For instance, the tempo of the song would increase, but the runner's pace would decrease. It became apparent that there was a mismatch between the perceived tempo of the song and the actual tempo. To better understand this phenomenon, a small user study was devised. With the study, we also intended to shed some light onto which music features have an impact on the perceived tempo of a song.

The study consisted of a web-page application that allowed the user to hear 30 second clips from 17 different songs selected from MPTrain's DML. The user was then asked to rank these songs in order of perceived speed, taking as much time as needed. Interestingly, none of the 8 study participants ${ }^{6}$ correctly placed the songs in their proper ordering based on tempo. However, there was some measure of consensus as to which songs seemed particularly fast or particularly slow. After analyzing the results we noted that approximately $25 \%$ of the songs were typically ranked substantially (i.e. greater than 2 positions away from the true ordering based on tempo) slower, or substantially faster than their actual tempo would dictate. We then analyzed these songs and discovered that they featured either many subdivisions per beat (faster) or very few subdivisions per beat (slower). This phenomenon was made especially clear by the presence of two clips from different parts of the same song ${ }^{7}$. The first clip was consistently perceived as being much faster than the actual tempo, whereas the second clip was considered to be much slower despite featuring the same core tempo. In the first clip there is a high degree of musical motion and many subdivisions per beat. On the other hand, the second clip plods along with many notes connecting together the major beats.

While the observation that subdivisions of the major tempo has a substantial impact of perceived speed is not surprising, this small study corroborates our experience with the participants in the running study. It also suggests that such a perception is shared between subjects. Therefore, it could be possible to add the perceived tempo to MPTrain's song metadata. We plan to do so in the next versions of the system.

5) Questionnaire Analysis: Table III summarizes our findings from the post-run questionnaires. The cell in bold high-

\footnotetext{
${ }^{6}$ They were different participants from the runners in the running study.

${ }^{7}$ Presidents of the United States of America - "Naked and Famous".
}

TABLE III

SUMMARY OF QUESTIONNAIRE ANSWERS

\begin{tabular}{|c|c|c|c|c|}
\hline & \multicolumn{4}{|c|}{ Running Condition } \\
\hline & Mute & Random & MPTrain & Metron. \\
\hline$>5$ miles $(\%)$ & 76.4 & 76.5 & 92.3 & 81.8 \\
\hline not cumbersome & 76.4 & 64.7 & 92.4 & 91.0 \\
\hline more energy & 76.4 & 94.1 & 76.9 & 91.0 \\
\hline more effective & 70.7 & 88.2 & 84.6 & 91.0 \\
\hline $\begin{array}{c}\text { more effective } \\
\text { towards workout goals }\end{array}$ & 64.7 & 64.7 & 84.6 & 91.0 \\
\hline equally or more enjoyable & 52.8 & 76.5 & 76.9 & 63.7 \\
\hline $\begin{array}{l}\text { more effective } \\
\text { within study }\end{array}$ & N/A & 29.4 & 76.9 & 77.8 \\
\hline $\begin{array}{l}\text { worked harder } \\
\text { within study }\end{array}$ & $\mathrm{N} / \mathrm{A}$ & 29.4 & 23.1 & 36.4 \\
\hline $\begin{array}{l}\text { more enjoyable } \\
\text { within study }\end{array}$ & N/A & 76.5 & 92.3 & 54.7 \\
\hline music increased enjoyment & N/A & 77.62 & 100 & 81.8 \\
\hline $\begin{array}{c}\text { music slightly } \\
\text { matched workout goals }\end{array}$ & N/A & 23.5 & 46.2 & 45.5 \\
\hline $\begin{array}{c}\text { music strongly } \\
\text { matched workout goals }\end{array}$ & N/A & 0.0 & 30.8 & 27.3 \\
\hline $\begin{array}{c}\text { music slightly } \\
\text { assisted in achieving goals }\end{array}$ & $\mathrm{N} / \mathrm{A}$ & 35.3 & 46.2 & 45.5 \\
\hline $\begin{array}{c}\text { music strongly } \\
\text { assisted in achieving goals }\end{array}$ & N/A & 0.0 & 38.5 & 36.4 \\
\hline
\end{tabular}

lights the running condition that scored highest for each of the questions.

A few interesting observations can be drawn from the Table:

1. Participants estimated that they ran for a longer distance in the MPTrain/Metronome condition than in the other conditions (first row on Table), even though the workout duration was exactly the same for all conditions.

2. They also found the system to be less cumbersome (second row on Table) when running on MPTrain/Metronome mode.

3. Participants found the workout to be more effective - both than average and within the study - and more enjoyable both than average and within the study - when running on MPTrain/Metronome mode than on any other mode (rows 4,5,7 and rows $6,9,10$ respectively).

4. Finally, participants found that the music as selected by MPTrain strongly matched the workout goals and assisted them in achieving those goals (rows 11 to 14).

\section{DISCUSSION AND FUTURE WORK}

A user study examined the use of physiological monitoring and auditory feedback to assist runners in achieving a predefined exercise goal. The study explored both quantitative and qualitative aspects of the system, and compared three different conditions: running without any audio, or on mute; running with randomly selected music, or on random, and running with music and/or a metronome as selected by the system, or on MPTrain/Metronome.

Both from a quantitative and qualitative perspective, running with auditory feedback (i.e. MPTrain/Metronome) was significantly superior to running on mute or on random modes:

- The MPTrain/Metronome condition enabled runners to achieve their workout goal for a higher percentage of the time than any other condition. 
- The Metronome mode performed best from a quantitative viewpoint, leading runners to spend an average of $62.3 \%$ of the time in the right heart rate zone.

- The MPTrain condition was the most enjoyable of all and increased the enjoyment of the run for $100 \%$ of the subjects.

- The Metronome condition was perceived as the most effective towards achieving the workout goals. However, the MPTrain condition was perceived as the one that best assisted runners in achieving the workout goals.

Our results confirm the hypotheses formulated in Section V-A.

In addition, the post-run questionnaires allowed participants to leave comments about the system and about each of the running conditions.

We shall highlight next a few of the most representative comments. Music was unanimously cited as the key factor that increased the enjoyment of the run. Runners enjoyed the selection that was automatically generated by MPTrain, despite the fact that it was a generic music library and not their personal collection. They appreciated the dynamic aspects of the system and the fact that the selection of music was done on-the-fly, depending on the context. They enjoyed knowing that the music was encouraging them to run faster, slower or at the same pace. In the words of one of the participants, "The music helped me keep a good pace. I got lost in the music and did not realize that I was doing physical activity. When a fast song came in, I knew that I had to run faster for just the duration of the song, before a new song would come in”.

It was also mentioned that the MPTrain condition provided better rhythm and a better paced workout than usual. Several participants suggested adding other forms of auditory feedback, including voice (e.g. encouraging words).

The most problematic aspect of the study, without a doubt, was the hardware. We experienced hardware problems with the heart rate monitor that were frustrating and slowed down the progress of the study. The connector to the headphones was also somewhat unreliable and produced interruptions in the audio at times. We plan on using a Bluetooth headset in the next versions of the system, as was suggested by multiple participants.

The study had a very positive impact on a significant portion of participants. Some of them asked us for the music library. Some others discovered that slower music was effective and enjoyable for the warming up and cooling down parts of the workout and planned on including that in their music collection.

The vast majority of participants considered it to be "a very fun study" and requested to be informed of further similar studies.

There are several lines of future research that we would like to pursue with the MPTrain system:

1) We are starting a long term runner study with a small number of runners, who will wear the MPTrain system for all their running sessions over a period of at least 4 weeks. We are interested in exploring how well runners do over time, as they get used to the system's style of coaching. The long term study will enable runners to select their own workout and to include songs from their personal music library. It will also incorporate information about the runner's past performance to the music selection algorithms.

2) We are working on incorporating new musical features to the music selection algorithms, such as the song's perceived tempo.

3) We are interested in incorporating additional contextual information, such as GPS data, body and external temperature, etc. MPTrain will use this information to produce better music selections.

4) We plan to test the system on other sports, such as skating and cyclying.

5) We plan to add a "rating" functionality to MPTrain's interface, such that users can very easily rate each song with respect to: (a) its effectiveness towards reaching the desired workout and (b) how much the user enjoys listening to it.

6) We are looking into various approaches to have users share their workout information (both in real-time and historic summaries) with friends and family. There are a number of interesting scenarios and new applications and services that we are considering in this direction.

7) Finally, we are working on different user interfaces to allow users to rate their workout, review their past workouts, and identify trends and deviations from those trends. We would also like to include lifestyle variables, such as diet, overall mood, stress levels, date of the workout, weather conditions, etc, and find correlations between them and the quality of the workouts.

\section{REFERENCES}

[1] N. Becker, S. Brett, C. Chambliss, K. Crowers, P. Haring, C. Marsh, and R. Montemayor, "Mellow and frenetic antecedent music during athletic performance of children, adults, and seniors," Percept. Motor Skills, vol. 79(2), pp. pp. 1043-1046, 1994

[2] L. Kravitz, "The effects of music on exercise?" IDEA Today, vol. 12(9), pp. pp. 56-61, 1994.

[3] W. Lee, "The effect of music on walking performance of older adults," Master's thesis, Ball State University, 2001.

[4] A. Beckett, "The effects of music on exercise as determined by physiological recovery heart rate and distance," J. of Music Therapy, vol. 27, pp. 126-136, 1990

[5] M. Anshel and D. Marisi, "Effects of music and rhythm on physical performance," Research Quaterly, vol. 49, pp. 109-112, 1979.

[6] J. Potteiger, J. Schroeder, and K. Goff, "Influence of music on ratings of perceived exertion during 20 minutes of moderate intensity exercise," Perceptual Motor Skills, vol. 91, pp. 848-854, 2000.

[7] T. Pujol and A. Langenfeld, "Influences of music on wingate anaerobic test performance," Perceptual Motor Skills, vol. 88(1), pp. 292-296, 1999.

[8] http://ipod.com, 2005

[9] Suunto, "T6, foot pod, n6hr," http://www.suunto.com.

[10] http://jogtunes.com, 2006

[11] I. running iTunes playlist, "http://www.lifehacker.com/software/running/intervalrunning-itunes-playlist-154491.php," 2006

[12] http://www.apple.com/ipod/nike, 2006.

[12] http://www.apple.com/ipod/nike,

[14] M. Staum, "Music and rhythmic stimuli in the rehabilitation of gait disorders," Journal of Music Therapy, vol. 20, pp. 69-87, 1983

[15] S. Stannard and M. Thompson, "Heart rate monitors: Coaches' frien of foe?" Sports Coach, vol. 21, pp. pp. 36-37, 1998.

[16] http://www.pnc.edu/hr/wellness/target_heart_rate_zone_training.htm, 2006.

[17] http://www.brianmac.demon.co.uk/hrm1.htm, 2006.

[18] W. Miller, J. Wallace, and K. Eggert, "Predicting max hr and the hr-vo2 relationship for exercise prescription in obesity," Medicine \& Science in Sports and Exercise, vol. 25(9), pp. pp. 1077-1081, 1993. 